\title{
EXERCÍCIO AERÓBIO REDUZ A HIPERTENSÃO ARTERIAL DE MULHERES COM DOENÇA DE CHAGAS
}

\author{
AEROBIC EXERCISE REDUCES HYPERTENSION IN WOMEN WITH CHAGAS DISEASE \\ EJERCICIO AERÓBICO REDUCE LA HIPERTENSIÓN ARTERIAL DE MUJERES CON MAL DE CHAGAS
}

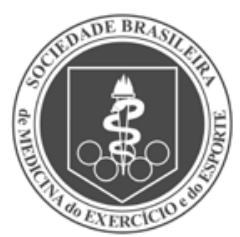

Artigo Original
Wania da Silva Lopes'

(Fisioterapeuta)

Roberto Kenji Nakamura Cuman ${ }^{1}$ (Farmacêutico)

Terezinha Aparecida Guedes (Matemática)

Silvana Marques de Araújo

(Farmacêutica)

Mônica Lúcia Gomes ${ }^{1}$

(Farmacêutica)

1. Universidade Estadual de

Maringá, Maringá, PR, Brasil.

\section{Correspondência:}

Mônica Lúcia Gomes,

Departamento de Ciências

Básicas da Saúde, Parasitologia,

Universidade Estadual de Maringá.

Av. Colombo, 5790, Zona Sete,

87020-900, Maringá, PR, Brasil.

mlgomes@uem.br

\begin{abstract}
RESUMO
Introdução: Os portadores de doença de Chagas frequentemente apresentam a hipertensão arterial sistêmica (HAS) como a principal comorbidade. Em indivíduos hipertensos com e sem doença de Chagas, o controle de HAS geralmente é medicamentoso. Medidas alternativas de intervenção como o exercício físico aeróbio têm sido preconizadas como a maneira mais efetiva para reduzir os níveis de pressão arterial. Objetivo: Avaliar a influência do exercício físico sobre a pressão arterial de mulheres hipertensas com e sem doença de Chagas. Métodos: Dezenove voluntárias divididas nos grupos G1 (nove com doença de Chagas) e G2 (dez sem doença de Chagas) foram submetidas a um programa de treinamento de 12 semanas, com duração de 30 a 60 minutos duas vezes por semana. A pressão arterial sistólica (PAS), diastólica (PAD) e a frequência cardíaca (FC) foram avaliadas no pré e pós-esforço no início (T0), após seis (T6) e 12 (T12) semanas. Resultados: Em T6, melhora significativa foi observada na PAS pré e pós-esforço e na PAD pós-esforço, para ambos os grupos. No T12, G1 apresentou melhora significativa para todas as variáveis, exceto FC pós-esforço e G2 para PAS pré e pós-esforço e FC pós-esforço. Não houve diferença significativa entre G1 e G2 para as variáveis estudadas. Conclusão: O exercício físico aeróbio de baixa intensidade reduz significativamente a pressão arterial de mulheres com doença de Chagas, pode ser realizado com segurança, e insere os pacientes com esta enfermidade na prática rotineira de exercícios.
\end{abstract}

Palavras-chave: hipertensão arterial, doença de Chagas, exercício físico, gênero feminino.

\begin{abstract}
Introduction: Patients with Chagas disease often have high blood pressure (HBP) as the main co-morbidity. In hypertensive individuals with and without Chagas disease, the control of hypertension is generally through medication. Alternative intervention measures such as aerobic exercise have been recommended as an effective way to reduce blood pressure levels. Objective: Assess the influence of exercise on blood pressure in hypertensive women with and without Chagas disease. Methods: Nineteen volunteers divided into two groups: G1 (nine with Chagas disease) and G2 (ten without Chagas disease) were submitted to a training program for 12 weeks, during 30 to 60 minutes, twice a week. The systolic (PAS) and diastolic (PAD) blood pressure and heart frequency (FC) were analyzed before and after the effort at the beginning (TO), after six weeks (T6) and twelve weeks (T12).. Results: In T6, significant improvement was observed in PAS pre-and post- effort and the PAD post-effort, for both groups. In T12, G1 showed significant improvement for all variables, except FC after effort and G2 for PAS before and after effort and FC after effort. There was no significant difference between G1 and G2 for all variables. Conclusion: Low-intensity aerobic exercise significantly reduces blood pressure in women with Chagas disease, can be performed safely and incorporate patients with this disease into the routine practice of exercises.
\end{abstract}

Keywords: arterial hypertension, Chagas disease, physical exercise, female gender.

\section{RESUMEN}

Introducción: Los portadores de Mal de Chagas frecuentemente presentan la hipertensión arterial sistémica (HAS) como la principal comorbidez. En individuos hipertensos con y sin Mal de Chagas el control de HAS generalmente es medicamentoso. Las medidas alternativas de intervención como el ejercicio físico aeróbico han sido preconizadas como la manera más efectiva para reducir los niveles de presión arterial. Objetivo: Evaluar la influencia del ejercicio físico sobre la presión arterial de mujeres hipertensas con y sin Mal de Chagas. Métodos: Diecinueve voluntarias divididas en los grupos G1 (nueve con Mal de Chagas) y G2 (diez sin Mal de Chagas) fueron sometidas a un programa de entrenamiento de 12 semanas, con duración de 30 a 60 minutos dos veces por semana. La presión arterial sistólica (PAS), diastólica (PAD) y la frecuencia cardíaca (FC) fueron evaluadas en el pre y post esfuerzo al inicio (TO), después de seis (T6) y 12 (T12) semanas. Resultados: En T6, mejora significativa fue observada en la PAS pre y post esfuerzo y en la PAD post esfuerzo, para ambos grupos. En el T12, G1 presentó mejora significativa para todas las variables, excepto FC post esfuerzo y G2 para PAS pre y post esfuerzo y FC post esfuerzo. No hubo diferencia significativa entre G1 y G2 para las variables estudiadas. Conclusión: El ejercicio físico aeróbico de baja intensidad reduce significativamente la presión arterial de mujeres con Mal de Chagas, puede ser realizado con seguridad e coloca a los pacientes con esta enfermedad en la práctica rutinaria de ejercicios.

Palabras clave: hipertensión arterial, mal de Chagas, ejercicio físico, género femenino. 


\section{INTRODUÇÃO}

Atualmente, apesar das medidas efetivas no combate a transmissão vetorial e transfusional do Trypanosoma cruzi, ainda existe uma quantidade expressiva de portadores da doença de Chagas tanto na América Latina, quanto no Brasil'. Recentemente, na região noroeste do estado do Paraná foi observado que dos pacientes atendidos em um centro de referência em saúde (Centro Regional de Especialidades de Maringá - CRE/PR) 6,8\% apresentaram sorologia positiva para o T. cruzi, indicando que nesta região existe um contingente de portadores crônicos desta infecção que necessitam de assistência adequada.

Dados na literatura demonstram que pacientes portadores de doença de Chagas, que apresentam idade um pouco mais avançada, têm predisposição para outras doenças crônicas ${ }^{3}$. Dentre estas doenças, a hipertensão arterial sistêmica (HAS) se destaca como a principal comorbidade ${ }^{3-7}$, embora haja estudos que mostram não haver diferença significante entre a frequência de HAS entre pacientes com e sem doença de Chagas ${ }^{4}$. Entretanto, Bozelli et al..$^{5}$ observaram que 25,3\% dos pacientes portadores de doença de Chagas atendidos no Hospital Universitário de Maringá, PR apresentavam também HAS. Gurgel et al. ${ }^{6}$ observaram que pacientes com doença de Chagas e HAS tiveram um comprometimento cardíaco maior.

Como a HAS está relacionada com a atividade do sistema nervoso simpático e com o funcionamento cardíaco, e ambos podem ser afetados pela infecção causada pelo T. cruzi, estudos buscando relacionar estas duas enfermidades crônicas têm sido realizados ${ }^{5,7}$.

Em indivíduos hipertensos com e sem doença de Chagas, o controle de HAS geralmente é medicamentoso. Atualmente, as medidas não farmacológicas de mudanças de hábitos de vida têm sido recomendadas na sua prevenção e controle ${ }^{8}$. Entre estas medidas se destacam o exercício físico, a redução do peso corporal e a redução de sódio na dieta 8 .

De acordo com a VI Diretriz Brasileira de Hipertensão Arterial ${ }^{8}$, a atividade física moderada de forma contínua ou acumulada, com duração de 30 a 60 minutos e frequência de três a cinco vezes por semana, deve ser realizada por todo adulto, desde que em condições de realizá-la, sendo uma maneira efetiva de reduzir os níveis de pressão arterial, manter uma boa saúde cardiovascular e qualidade de vida. O exercício físico pode controlar a hipertensão leve e quando associado ao tratamento farmacológico pode melhorar a hipertensão grave e moderada8.

Raros são os estudos que envolvem doença de Chagas e exercício físico ${ }^{9,10}$. Nestas pesquisas foi observado nestes pacientes melhora da capacidade funcional aeróbia por meio de alterações na composição corporal e perfil lipídico, promovendo diminuição de riscos de complicações cardiovasculares e superior capacidade funcional de pacientes em fase inicial da cardiopatia chagásica em relação àqueles em fase avançada. Em nossa pesquisa observamos que o exercício físico aeróbio aumentou a tolerância ao esforço em pacientes com doença de Chagas crônica e que este pode ser realizado com segurança"1.

A maioria dos estudos que envolvem o exercício físico é realizada com indivíduos do gênero masculino ${ }^{12}$. Na última década, a população feminina também passou a ser estudada. O gênero feminino apresenta maior percepção de doenças, maior tendência para o autocuidado e busca assistência médica com maior frequência. ${ }^{13,14}$ As mulheres também apresentam maior conhecimento sobre o papel do exercício físico na prevenção e no tratamento da hipertensão ${ }^{15}$, indicando que pode ocorrer melhor adesão a programas de treinamento por este gênero. Neste trabalho foi proposto verificar a influência do exercício físico aeróbio sobre a pressão arterial e frequência cardíaca de mulheres hipertensas com e sem doença de Chagas.

\section{MATERIAIS E MÉTODOS}

Cada participante foi esclarecida quanto ao propósito do estudo e assinou um termo de consentimento aprovado pelo Comitê de Ética em Pesquisa com Seres Humanos da Universidade Estadual de Maringá, sendo garantida a proteção da privacidade dos voluntários, conforme determinações das Diretrizes e Normas Regulamentadoras de Pesquisas Envolvendo Seres Humanos do Conselho Nacional de Saúde $\left(n^{\circ}\right.$ 196/96) ${ }^{16}$.

Participaram deste estudo 19 mulheres hipertensas que foram avaliadas clinicamente por um médico cardiologista, o qual verificou o grau da hipertensão arterial, avaliou as alterações cardíacas através do eletrocardiograma e autorizou a participação neste estudo. Em seguida as participantes foram encaminhadas ao Laboratório de Doença de Chagas da Universidade Estadual de Maringá (LDC/UEM) onde realizavam exame sorológico de imunofluorescência indireta para doença de Chagas. Todas as voluntárias foram acompanhadas ao setor de cardiologia do Centro Regional de Especialidades da região Noroeste do Paraná (CRE/PR) no período de janeiro a dezembro de 2009, e por meio dos prontuários obtivemos outros dados clínicos e laboratoriais. As voluntárias portadoras de hipertensão controlavam a pressão arterial pelo uso de inibidores da enzima conversora de angiotensina (captopril), diuréticos (hidroclorotiazida) e betabloqueadores (atenolol e propanolol). As participantes foram divididas em dois grupos pareados de acordo com a faixa etária. O primeiro grupo (G1) contendo nove participantes com doença de Chagas e o outro (G2) contendo 10 participantes sem doença de Chagas. A alocação em cada grupo foi feita de forma aleatória, de acordo com a aptidão ao exercício físico e após avaliação clínica. As características físicas e antropométricas, assim como a classe funcional das participantes não foram significativamente diferentes (tabela 1).

Critérios de Inclusão: apresentar hipertensão leve (PAS 140 a 159 mmHg e PAD 90 a 99 mmHg), ter ou não doença de Chagas, gênero feminino, faixa etária entre 45 e 69 anos, classe I da New York Heart Association (NYHA), aptidão ao exercício físico, disponibilidade e interesse para participar do exercício físico durante 12 semanas, duas vezes por semana.

Critérios de Exclusão: hipertensão grave, diagnóstico de bloqueio completo do ramo direito, arritmias ventriculares diversas, extra-sístoles ventriculares polimorfas, taquicardia ventricular, bloqueio atrioventricular (segundo e terceiro grau), insuficiência coronariana, diabetes, patologias musculoesqueléticas que impediriam a prática do protocolo, doenças pulmonares incapacitantes, doenças endócrinas e insuficiência cardíaca classe II, III e IV, frequência de comparecimento nos treinos inferior a duas vezes por semana e comparecimento no programa por um período inferior a dez semanas.

Tabela 1. Características físicas e classe funcional de mulheres hipertensas com e sem doença de Chagas.

\begin{tabular}{c|c|c|c}
\hline \multirow{2}{*}{ Variáveis } & $\begin{array}{c}\text { Com doença de } \\
\text { Chagas (G1) }\end{array}$ & $\begin{array}{c}\text { Sem doença de } \\
\text { Chagas (G2) }\end{array}$ & \\
\cline { 2 - 4 } & $\mathbf{n = 9}$ & $\mathbf{n}=\mathbf{1 0}$ & $\mathbf{p}$ \\
\hline Idade (anos)* & $61,4 \pm 5,9$ & $58,4 \pm 7,8$ & 0,355882 \\
\hline Estatura (m)* & $1,58 \pm 0,07$ & $1,57 \pm 0,05$ & 0,675559 \\
\hline Peso inicial (T0) (kg) & $71,5 \pm 12,2$ & $68,1 \pm 10,6$ & 0,522050 \\
\hline Peso final (T12) (kg) & $70,7 \pm 12,0$ & $67,9 \pm 10,5$ & 0,598177 \\
\hline Classe Funcional (NYHA) & । & । & \\
\hline
\end{tabular}

* Média \pm desvio padrăo; NYHA: New York Heart Association. 
A pressão arterial sistólica (PAS), pressão arterial diastólica (PAD) e frequência cardíaca (FC) foram avaliadas antes e após cada sessão do programa, o que corresponde ao pré e pós-esforço. A PAS e PAD foram aferidas no braço esquerdo das pacientes, na posição sentada, pelo método indireto e técnica auscultatória utilizando um estetoscópio e um esfigmomanômetro da marca BD previamente calibrado. A FC foi medida por técnica manual, pela palpação da artéria radial por um único avaliador. As variáveis foram medidas no T0 (início do estudo), no T6 (após seis semanas) e no T12 (após 12 semanas).

As pacientes acompanhados clinicamente no CRE/PR realizaram um programa de exercício físico. Como o teste ergométrico não foi aplicado, a intensidade do exercício foi estabelecida pela respiração e pelo cansaço subjetivo das voluntárias, onde cada participante deveria permanecer discretamente ofegante, conseguindo falar frases completas, sem interrupções, sentindo-se moderadamente cansada. Mesmo não sendo um dos métodos mais precisos, este protocolo é previsto pela VI Diretriz Brasileira de Hipertensão (2010)8. Como não há nada estabelecido nesta área para pacientes com doença de Chagas, utilizamos um protocolo para pacientes com cardiopatias de outras etiologias. Para a prescrição do exercício os beta-bloqueadores não foram considerados, pois de acordo com alguns autores ${ }^{17,18}$ esta medicação não exerceu influência sobre os benefícios do treinamento físico.

Para a realização do programa, as participantes foram alocadas em grupos menores contendo três ou quatro participantes com e sem doença de Chagas. O protocolo teve duração de doze semanas, com frequência de duas vezes por semana em dias alternados, com aumento gradual a cada sessão até atingir o limite máximo de uma hora de duração, considerando as condições clínicas da paciente. O seguinte programa de treinamento físico foi aplicado: inicialmente foi realizado o aquecimento muscular com alongamentos ativos de membros superiores e inferiores, com duração de 5 minutos, progredindo para 10 minutos na terceira sessão de treinamento; em seguida foi realizada a fase aeróbia do exercício por meio de caminhada iniciando com 15 minutos, progredindo para 20 minutos na segunda sessão e chegando a 30 minutos na terceira sessão; ao final de cada sessão foi realizado o resfriamento para recuperação seguido do relaxamento, sendo que cada uma dessas fases iniciaram com 5 minutos e progrediram para 10 minutos na terceira sessão.

O programa de exercício físico foi realizado no período matutino, em local aberto, plano e sombreado.

\section{Análise Estatística}

Foi realizada utilizando o programa Statistica 7.0. As variáveis estudadas foram expressas na forma de média e desvio padrão. Foram aplicados os testes Lillefors, Kolmogorov-Smirnov e Shapiro-Wilk's W e com base neles foi verificado que havia médias com e sem padrão de normalidade o que nos levou a realizar a análise por meio de testes não paramétricos. Para determinar as diferenças intragrupos nos tempos T0, T6 e T12 foi utilizado o teste de Wilcoxon e para comparar as diferenças entre os grupos G1 e G2 foi utilizado o teste de Mann-Whitney. O nível de significância foi de 5\%.

\section{RESULTADOS}

Todas as voluntárias submetidas ao programa de treinamento físico faziam uso de medicação para controlar a hipertensão. Para os grupos G1 e G2 foram utilizados, respectivamente, inibidores de enzima conversora de angiotensina (44,4\% e 60\%), betabloqueadores (33,3\% e 30\%) e diuréticos (22,2\% e 10\%). Nesse estudo apenas seis $(31,6 \%)$ pacientes utilizaram betabloqueadores: três do grupo G1 e três do G2. A análise dos dados de pacientes que faziam ou não o uso desta droga demonstrou que a única diferença significativa ( $p=0,0436)$ foi na FC pré-esforço após 12 semanas, indicando que os resultados dos grupos avaliados não sofreu interferência do uso do betabloqueador.

A avaliação da pressão arterial sistólica, diastólica e da frequência cardíaca das participantes com doença de Chagas (G1) e sem doença de Chagas (G2) no tempo T0, T6 e T12, pode ser observada nas figuras 1, 2 e 3, respectivamente. Após seis semanas de exercício físico, a comparação entre as participantes do grupo G1 mostrou diferenças significativas na PAS pré $(p=0,0133)$ e pós-esforço $(p=0,0076)$ e na PAD pós-esforço $(p=0,0431)$. Em $G 2$, estas mesmas variáveis apresentaram diferenças significativas PAS pré $(p=0,0277)$ e pós-esforço $(p=0,0179)$ e PAD pós-esforço $(0,0431)$.

Após 12 semanas de exercício físico, em G1 houve redução na PAS de $20 \mathrm{mmHg}(p=0,0076)$ no pré-esforço (figura $1 \mathrm{~A})$, e de $24 \mathrm{mmHg}$ no pós-esforço $(0,0076)$ (figura 1B). Em G2 reduções de $7 \mathrm{mmHg}(p=0.0431)$ foram observadas na PAS pré e de $8 \mathrm{mmHg}(p=0,0277)$ no pós-esforço (figuras $1 A$ e B). Para as participantes do grupo G1 houve uma diminuição na PAD de 12,2 mmHg ( $p=0,0179$ ) no pré-esforço (figura 2A) e de $10 \mathrm{mmHg}(0,0179)$ no pós-esforço (figura 2B). Diferenças significativas foram observadas para FC pré-esforço ( $p=0,0208)$ em $G 1$ (figura 3A) e FC pós-esforço ( $p=0,0050)$ em G2 (figura 3B).

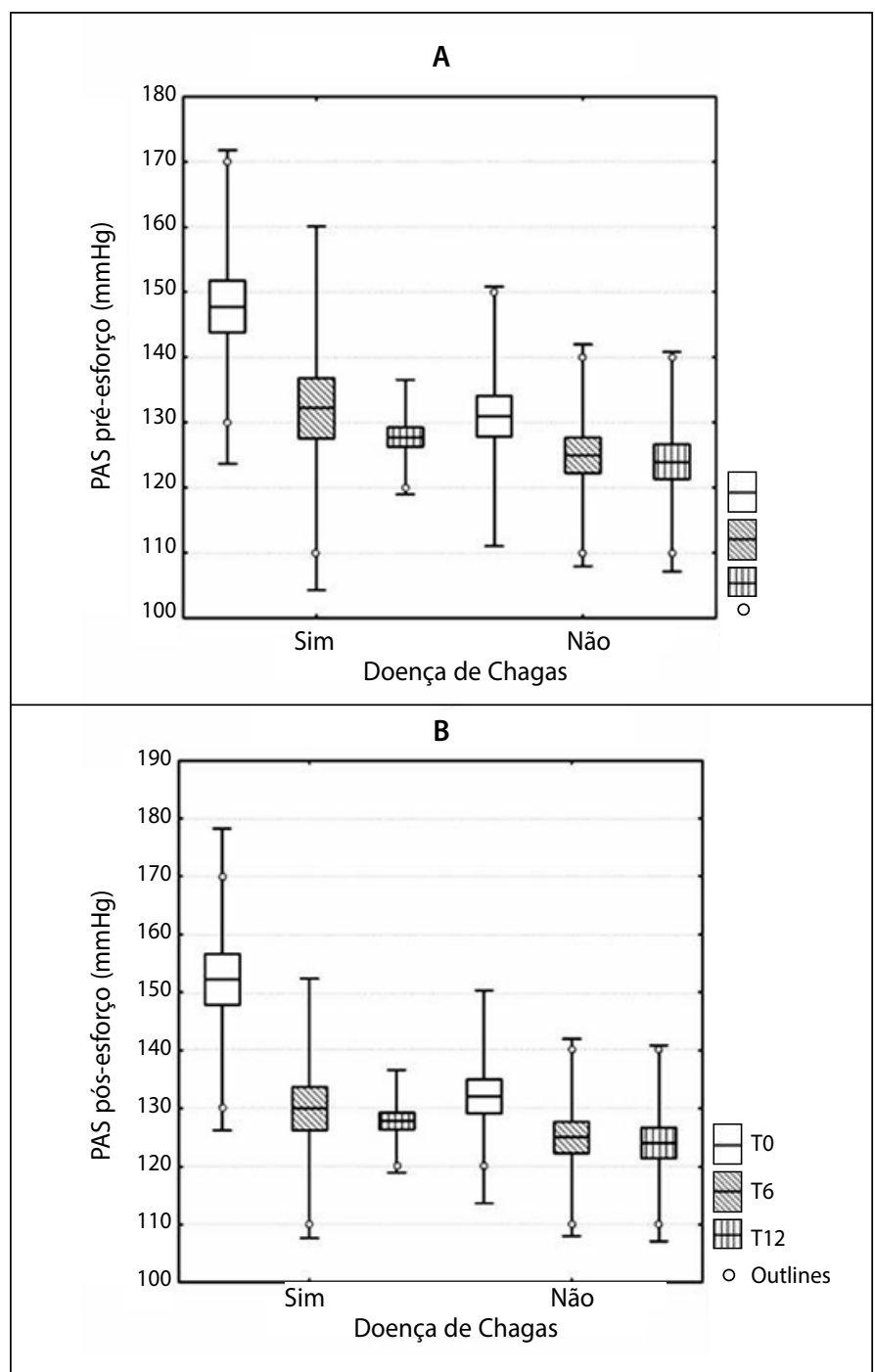

Figura 1. Pressão arterial sistólica pré-esforço (A) e pós esforço (B) entre T0 (início do estudo), T6 (após 6 semanas) e T12 (após 12 semanas) em mulheres com (G1) e sem doença de Chagas (G2). 


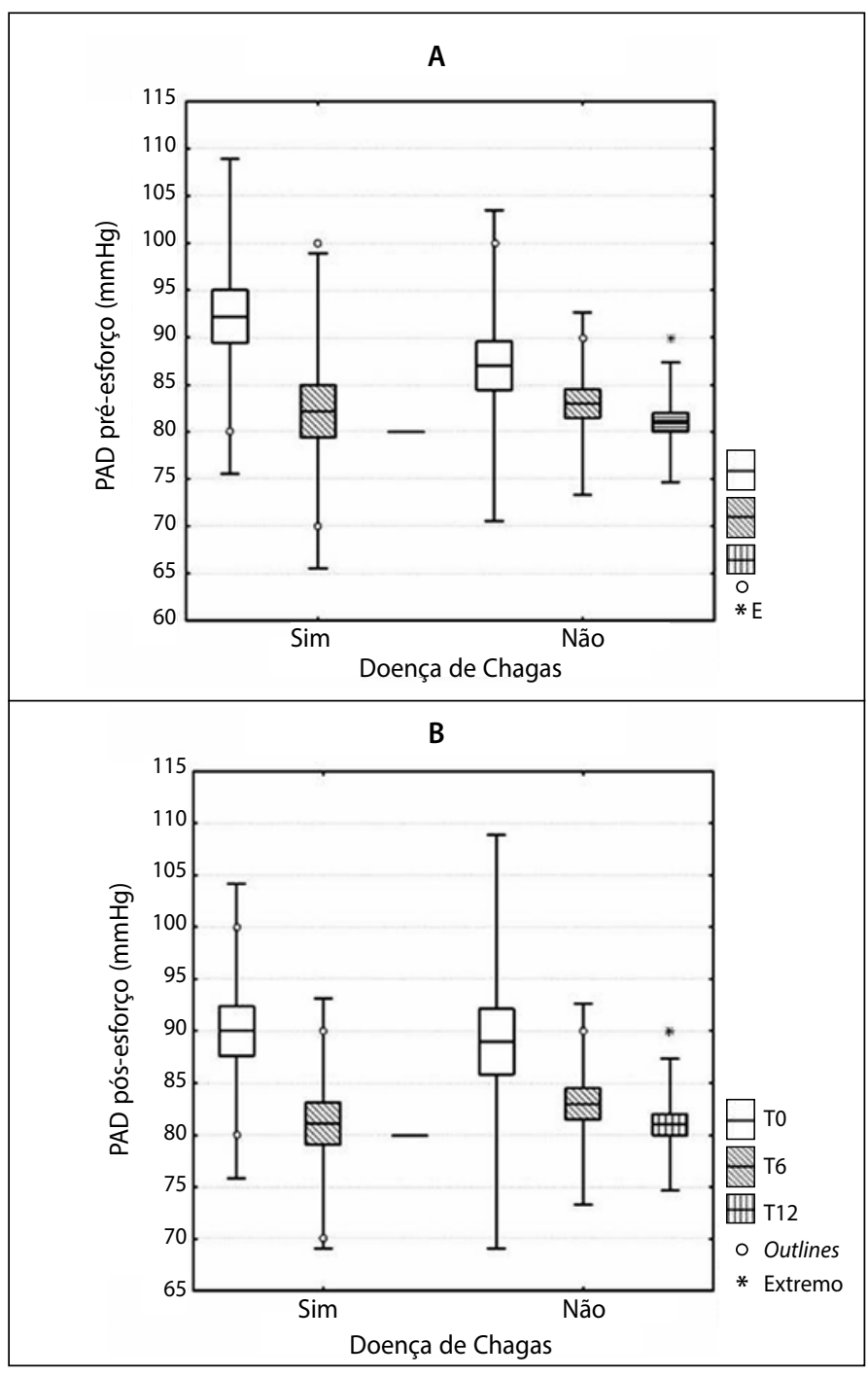

Figura 2. Pressão arterial diastólica pré-esforço (A) e pós esforço (B) entre T0 (início do estudo), T6 (após 6 semanas) e T12 (após 12 semanas) em mulheres com e sem doença de Chagas.

Comparando os grupos G1 e G2 no T0, T6 e T12, diferença significativa foi observada somente no T0 para as variáveis PAS pré $(p=0,0089)$ e pós-esforço $(p=0,0032)$.

Ao longo do programa não houve desistência de nenhum dos participantes e nem intercorrências que justificassem sua interrupção.

\section{DISCUSSÃO}

Vários autores ${ }^{3-7}$ mostram que HAS é a comorbidade mais frequente entre os indivíduos com doença de Chagas, mas estudos que envolvam estas doenças e exercícios físicos não têm sido relatados. Neste trabalho foi realizado um programa de treinamento, com base em protocolo aplicado a pacientes com cardiopatias de outras etiologias, ${ }^{8}$ já que nada tem sido estabelecido nesta área para pacientes com doença de Chagas. Neste sentido, o teste ergométrico não foi aplicado para determinar a intensidade do exercício, uma vez que primamos em estabelecer um protocolo simples e viável que possa inclusive ser utilizado em maior escala, como no Sistema único de Saúde. Optamos por um protocolo com sessões de até 60 minutos, duas vezes por semana, durante 12 semanas. De acordo com vários autores, ${ }^{19-22} \mathrm{o}$ aumento do tempo de exercício não proporciona maior redução da PAS e quedas pressóricas já ocorrem a partir da primeira sessão do exercício, indicando que programas de duas a cinco vezes por semana têm efeito hipotensor similar. Melhora

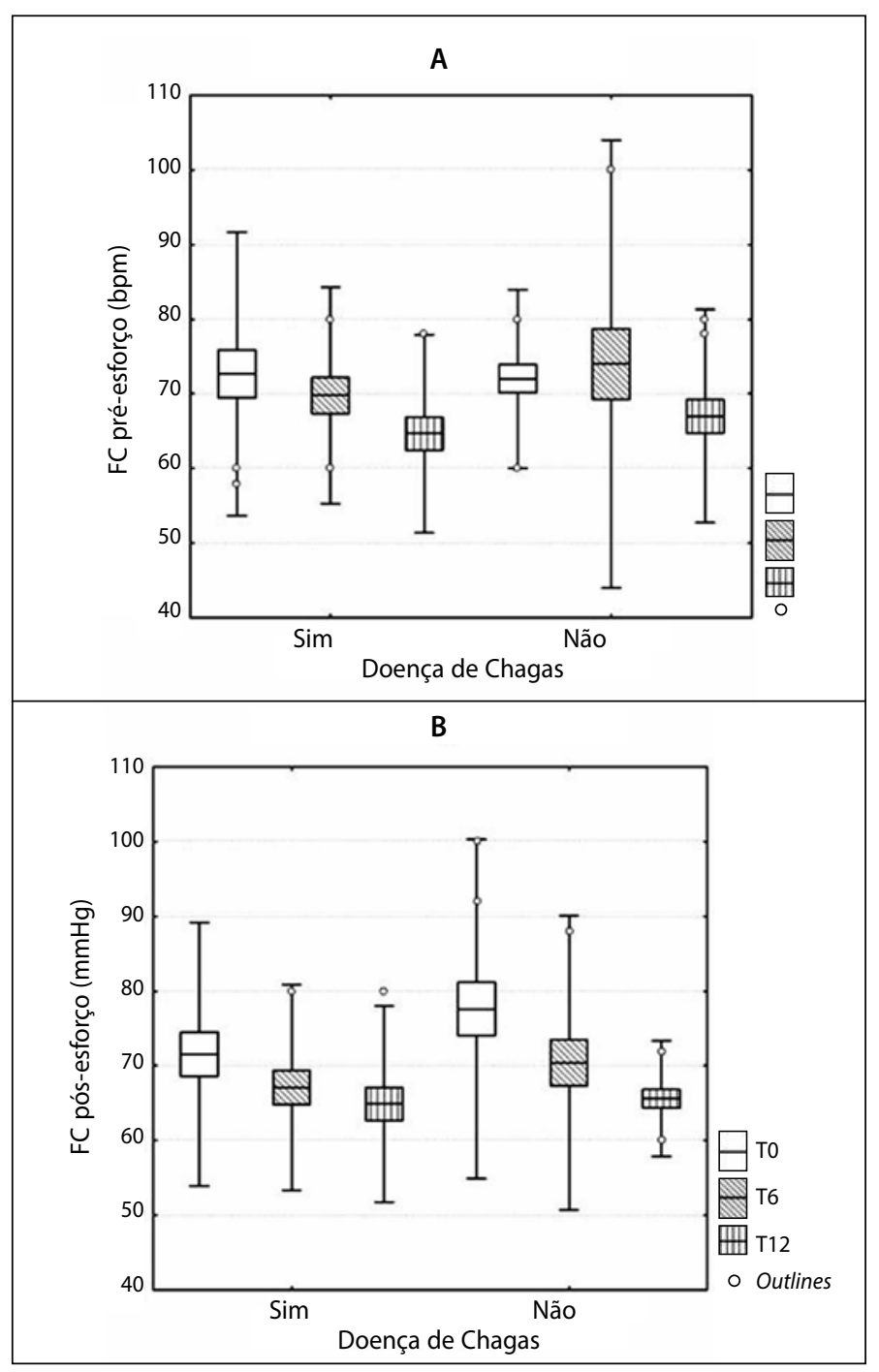

Figura 3. rrequëncia cardiaca pré-estorço (A) e pós estorço (B) entre TO (início do estudo), T6 (após 6 semanas) e T12 (após 12 semanas) em mulheres com e sem doença de Chagas.

significante nas pressões sistólica e diastólica das participantes foi observada, tanto com seis, quanto com 12 semanas de treinamento físico. Quando as participantes de cada grupo foram comparadas entre si, no T6 em relação ao T0, três de seis variáveis estudadas foram significativamente diferentes e no T12, cinco variáveis foram estatisticamente diferentes em G1 e três em G2. A comparação entre os grupos não mostrou diferenças significativas para todas as variáveis nos tempos T6 e T12.

Com seis semanas de exercício físico foi observada uma redução significativa na PAS pré e pós-esforço e na PAD pós-esforço, tanto para G1, quanto para G2. Estes resultados obtidos com um curto tempo de treinamento ${ }^{19}$ estão de acordo com vários autores que também observaram redução da PAS e PAD em programas que variaram de seis a dez semanas de duração 20,21 .

Os resultados observados com 12 semanas em participantes de G1 e de G2 estão de acordo com outros autores que realizaram programa de treinamento com este mesmo tempo de duração, 19-24 ou com 16 a 24 semanas $^{25,26}$. A redução significativa no pós-esforço da PAS em 24,4 mmHg e PAD em $10 \mathrm{mmHg}$ para G1 e de apenas $8 \mathrm{mmHg}$ na PAS em G2 é semelhante à queda pressórica de $15 \mathrm{mmHg}$ na PAS e $7 \mathrm{mmHg}$ na PAD observada por Viecilli et al. ${ }^{24}$. Este resultado tem importância clínica e demonstra que o exercício físico exerceu um efeito benéfico mais evidente nas participantes com doença de 
Chagas (G1). Estima-se que reduções de apenas $2 \mathrm{mmHg}$ na PAD podem diminuir substancialmente o risco de doenças e mortes associadas à hipertensão, demonstrando que a prática do exercício físico representa importante benefício para a saúde dos hipertensos ${ }^{27}$. Este resultado pode estar relacionado ao fato das participantes de $\mathrm{G} 1$ terem demonstrado uma maior motivação ao realizarem o exercício físico e maior preocupação com a sua saúde em função de apresentarem a doença de Chagas associada à hipertensão. Aqui cabe ressaltar a importância de se esclarecer e dar apoio ao paciente portador desta doença como preconizado pelo Programa $\mathrm{ACHE}^{28}$. Os resultados indicam ainda que o fato das participantes de G1 terem um diagnóstico de doença de Chagas não as limitou de realizar exercício físico, contribuindo para modificar o estigma que o paciente portador desta doença tem em relação ao seu diagnóstico ${ }^{28,29}$ e para auxiliar no controle da HAS. Mendes et al. ${ }^{11}$ também observaram que o treinamento aeróbio de curto período, de simples aplicação e com supervisão pode ser realizado com segurança por mulheres com doença de Chagas crônica da classe I da NYHA.

Doze semanas de exercício físico exerceu um efeito diferente sobre a FC de G1 e de G2. Em G1, diferença significativa foi observada na FC pré-esforço e em G2 na FC pós-esforço. Embora as participantes com doença de Chagas tenham respondido positivamente ao exercício físico no pré-esforço, esta resposta não ocorreu no pós-esforço. Este resultado pode estar relacionado ao fato de pacientes com insuficiência cardíaca de etiologia chagásica apresentar uma média de FC significativamente menor do que pacientes sem doença de Chagas, mesmo após ajuste com terapia medicamentosa, o que pode ser o reflexo da maior frequência de disfunção sinusal entre os chagásicos ${ }^{30,31}$. Além disso, sabe-se que os pacientes com insuficiência cardíaca têm um aumento na atividade simpática, decorrente de níveis elevados de catecolaminas circulantes, que parece estar associada à dessensibilização, redução dos receptores $\beta$ - adrenérgicos e baixa regulação dos receptores cardíacos e resposta inotrópica e cronotrópica deprimidas $^{32}$. O uso de betabloqueadores parece não ter influenciado os resultados da FC, uma vez que a única diferença significativa observada entre os pacientes que utilizavam ou não esta medicação foi na FC pré-esforço após 12 semanas de exercício físico, indicando que a melhora no desempenho das pacientes não teve relação com a medicação utilizada.

Diferenças significativas entre G1 e G2 não foram observadas para as variáveis estudadas, indicando que independente da participante ter ou não doença de Chagas, o exercício físico exerceu o mesmo efeito nos tempos estudados (T6 e T12). Estes resultados reforçam o fato de que as participantes que tem doença de Chagas podem realizar exercícios físicos com segurança, desde que estes sejam orientados por um profissional especializado, o que proporciona uma redução da pressão arterial, aumento da tolerância ao esforço físico e melhora da capacidade funcional ${ }^{11,33}$. Estes resultados também indicam que a concomitância de HAS e doença de Chagas não alteram a história natural de ambas as doenças como relatado por Gurgel et al. ${ }^{6}$ e que a interação entre elas não influencia o efeito de abordagens alternativas de intervenção, como o exercício físico.

Outro resultado do presente trabalho, que reforça a ação benéfica do treinamento proposto sobre a pressão arterial, é que mesmo as pacientes com doença de Chagas, apresentando diferença significativa da PAS pré e pós-esforço no T0 em relação às pacientes sem doença de Chagas, esta diferença não foi observada após 6 e 12 semanas. A PAS mais elevada no grupo de pacientes com doença de Chagas pode estar relacionada a própria fisiopatologia desta tripanossomíase, uma vez que a infecção pelo T. cruzi acomete as terminações nervosas parassimpáticas e pode acarretar uma desregulação neurovegetativa com predomínio do tônus simpático ${ }^{6}$.

Evidências científicas sobre a importância do exercício físico tanto para prevenção, quanto para retardo do aparecimento das doenças crônicas são cada vez mais frequentes e a efetividade de um programa de exercício físico na redução da pressão arterial já está bem estabelecida, mesmo em indivíduos que estão em tratamento farmacológico para hipertensão, como os indivíduos que participaram deste estudo e em outros $^{15,22-24,26,34-38}$. No entanto, os níveis de exercício físico em que os benefícios de prevenção poderiam ser potencializados ainda são muito baixos $^{27}$. Desta forma, o treinamento de baixa e moderada intensidade, associado ao exercício aeróbio, podem ser utilizados como tratamento não farmacológico em indivíduos hipertensos, acarretando diminuição da dose ou até mesmo a suspensão da medicação, reduzindo os efeitos colaterais do tratamento medicamentoso e o ônus para os órgãos de saúde pública $33,34,39$.

O efeito hipotensor do exercício físico de baixa intensidade observado no presente trabalho pode ser justificado pela diminuição no débito cardíaco que está associado ao decréscimo da frequência cardíaca de repouso e diminuição do tônus simpático no coração, em decorrência de menor intensificação simpática e maior retirada vagal ${ }^{40}$.

Entre as limitações deste estudo inclui-se a ausência de um teste de esforço para determinar a intensidade do treinamento físico, e o fato de não ter sido considerado o efeito dos betabloqueadores sobre a frequência cardíaca dos voluntários, porque os resultados para os grupos investigados não foram afetados pela utilização desta droga. O estudo foi realizado com o objetivo de desenvolver protocolos que fossem mais viáveis e aplicáveis em unidades de saúde pertencentes ao sistema nacional de saúde do Ministério da Saúde (Sistema Único de Saúde do Ministério de Saúde do Brasil - SUS/MS). Os resultados obtidos são promissores, pois, mesmo com estas limitações, o estudo demonstrou que o exercício físico aeróbio teve efeitos benéficos, reduzindo significativamente a PAS, PAD e FC de mulheres com e sem doença de Chagas.

\section{CONCLUSÕES}

Conclui-se neste estudo que o exercício físico aeróbio de baixa intensidade teve um efeito benéfico reduzindo significativamente a PAS, PAD e FC de mulheres com e sem doença de Chagas. Os resultados obtidos indicam que um programa de treinamento simples, de fácil aplicação, de baixo custo, que não necessita de habilidades especiais ou aprendizado pode ser realizado com segurança em pacientes com doença de Chagas crônica classe I da NYHA e como estratégia para prevenção e tratamento de HAS, proporcionando uma melhora na saúde dos hipertensos e retirando da condição de repouso indivíduos que têm doença cardíaca crônica, como a doença de Chagas, pois atualmente, um dos maiores desafios consiste em dar assistência adequada aos portadores crônicos desta infecção.

\section{AGRADECIMENTOS}

Os autores agradecem à Fundação Araucária (Apoio ao Desenvolvimento Científico e Tecnológico do Paraná), ao Conselho Nacional de Desenvolvimento Científico e Tecnológico (CNPq) e ao Departamento de Ciência e Tecnologia da Secretaria de Ciência, Tecnologia e Insumos Estratégicos (Decit/SCTIE) do Ministério da Saúde do Brasil pelo financiamento deste estudo.

Todos os autores declararam não haver qualquer potencial conflito de interesses referente a este artigo. 


\section{REFERÊNCIAS}

1. WHO (World Health Organization): New global effort to eliminate Chagas disease. Wkly Epidem Rec 2007;82(28-29):259-60

2. Lopes WS, Guedes TA, Araújo SM, Gomes ML. Hipertensão arterial: perfil sócio-demográfico e co-morbidades de pacientes da região sul do Brasil. Acta Sci Health Sci. 2012;34:119-26.

3. Alves RMA, Thoma RP, Almeia EA, Wanderley JS, Guariento ME. Doença de Chagas e envelhecimento: a associação de outras enfermidades crônicas em pacientes idosos chagásicos. Rev Soc Bras Med Trop. 2009;42(6):622-8

4. Almeida EA, Neto RMB, Guariento ME, Wanderley JS, Souza ML. Apresentação clínica da doença de Chagas crônica em indivíduos idosos. Rev Soc Bras Med Trop. 2007;40(3):311-5.

5. Bozelli CE, Araújo SM, Falavigna-Guilherme AL, Gomes ML. Perfil clínico-epidemiológico de pacientes $\mathrm{cm}$ doença de Chagas no Hospital Universitário de Maringá, Paraná, Brasil. Cad Saúde Pública. 2006;22(5):1027-34

6. Gurgel CBFM, Almeida EA. Frequência da hipertensão arterial em chagásicos crônicos e sua repercussão no coração: estudo clínico e anatomopatológico. Arq Bras Cardiol. 2007:89(3):191-200.

7. Bertanha L, Guariento ML, Magna LA, Almeida EA. Caracterização clínico-laboratorial de chagásicos hipertensos sem insuficiência cardíaca manifesta. Rev Soc Bras Med Trop. 2008;41(2):163-8,

8. Sociedade Brasileira de Cardiologia. Sociedade Brasileira de Hipertensão Arterial e Sociedade Brasileira de Nefrologia. VI Diretrizes Brasileiras de Hipertensão Arterial. Arq Bras Cardiol. 2010;95(1, Supl 1):1-51.

9. Oliveira FP, Pedrosa RC, Giannella-Neto A. Trocas gasosas em exercício em diferentes estágios evolutivos da cardiopatia chagásica crônica. Arq Bras Cardiol. 2000; 75(6):481-9.

10. Costa LM, Santos D, Oliveira DM, Neiva CM. Efeitos do exercício físico regular sobre o perfil bioquímico e a capacidade aeróbia máxima na doença de Chagas. Revista Cientifica da Universidade de Franca. 2007;7(1/3):109-15

11. Mendes MFA, Lopes WS, Wilson A, Nogueira GA, Araújo SM, Gomes ML. Atividade física aeróbia em mulheres com doença de Chagas. Fisioter Mov. 2011;24(4):591-60.

12. Lopes PP, Vanderlei LCM, Tarumoto MH. Perfil dos indivíduos frequentadores de programas ambulatoriais de exercícios físicos para pacientes cardíacos da cidade de Presidente Prudente (SP). Rev Soc Cardiol do Estado de São Paulo. 2005;3(Supl A): 19-28.

13. Zaitune MPA, Barros MBA, César CLG, Carandina L, Goldbaum M. Hipertensão arterial em idosos: prevalência, fatores associados e práticas de controle no município de Campinas, São Paulo, Brasi . Cad Saúde Pública. 2006;22(2):285-94.

14. Knuth AG, Bielemann RM, Shana GS, Borges TT, Del Duca GF, Kremer MM et al. Conhecimento de adultos sobre o papel da atividade física na prevenção e tratamento de diabetes e hipertensão: estudo de base populacional no sul do Brasil. Cad. Saúde Pública 2009;25(3):513-20.

15. Beauvais F. Physical training with beta-blockers in chronic heart failure, Ann Cardiol Angeiol. (Paris). 2010;59(4):229-33.

16. Conselho Nacional de Saúde (no 196/96) disponível na internet (http://conselho.saude.gov.br/docs/ Resolucoes/Reso196de96.doc).

17. Racine N, Blanchet M, Ducharme A, Marquis J, Boucher JM, Juneau M, White M., Decreased heart rate recovery after exercise in patients with congestive heart failure: effect of beta-blocker therapy. Card Fail. 2003:9(4):296-302.

18. Silva MSV, Bocchi EA, Guimarães GV, Padovani CR, Silva MHGG, Pereira SF, Fontes RD. Benefício do treinamento físico no tratamento da insuficiência cardíaca, estudo com grupo controle. Arq Bras Cardiol. 2002;79(4):351-6.

19. Pinto A, Raimondo DD, Tuttolomondo A, Fernandez P, Arne V, Licata G. Twenth-four hour ambulatory blood pressure monitoring to evaluate effects on blood pressure of physical activity in hypertensive patients. Clin J Sports Med. 2006;16(3):238-43.

20. Ishikawa-Takata KI, Ohta T, Tanaka H. How much exercise is required to reduce blood pressure in essential hypertensives: a dose-response study. Am J Hypertens. 2003;16(8):629-33.
21. Martinelli B, Barrile SR, Arca EA, Franco EA, Franco RJS, Cuadrado L. Influência do exercício aeróbio na renina de portadores de hipertensão arterial com sobrepeso. Arq Bras Cardiol. 2010;95(1):91-8.

22. Farinatti PTV, Oliveira RB, Pinto VLM, Monteiro WD, Francischetti E. Programa domiciliar de exercícios: efeito de curto prazo sobre a aptidão física e pressão arterial de indivíduos hipertensos. Arq Bras Cardiol. 2005;84(6):473-9.

23. Bundchen DC, Panigas CF, Dipp T, Panigas TF, Richter CM, Belli KC et al. Ausência de influência da massa corporal na redução da pressão arterial pós exercício. Arq Bras Cardiol. 2010;94(5):678-83.

24. Viecili PRN, Bundchen DC, Richter CM, Dipp T, Lamberti DB, Pereira AMR, et al. Curva dose-resposta do exercício em hipertensos: Análise do número de sessões para efeito hipotensor. Arq Bras Cardiol. 2009;92(5):393-9.

25. Nunes $A P O B$, Rios ACS, Cunha GA, Barreto ACP, Negrão CE. Efeitos de um programa de exercício físico não supervisionado e acompanhado a distância, via internet, sobre a pressão arterial e composição corporal em indivíduos normotensos e pré-hipertensos. Arq Bras Cardiol. 2006;86(4):289-96.

26. Ciolac EG, Guimarães GV. Exercício físico e síndrome metabólica. Rev Soc Bras Med do Esporte. 2004;10(4):319-23.

27. Araújo SM, Ando MH, Cassarotti DJ, Mota DCGA, Borges S, Gomes ML. Programa ACHEl: Atenção ao chagásico com Educação Integral no município de Maringá e região noroeste do Paraná, Brasil. Rev Soc Bras Med Trop. 2000;33(6):565-72

28. Mota DCGA, Pereira AMTB, Gomes ML, Araújo SM. Estresse e Resiliência em Doença de Chagas. Aletheia (Canoas). 2006;24:57-68.

29. Braga JCV, Reis F, Aras R, Costa ND, Bastos C, Silva R, Soares A, Júnior AM, Ásfora S, Latado AL. Aspectos clínicos e terapêuticos da insuficiência cardíaca por doença de Chagas. Arq Bras Cardiol. 2006;86(4):297-302

30. Braga AMFW, Rondon MUPB, Negrão CE, Wajngarten M. Predictive Value of Ventilatory and Metabolic Variables for Risk of Death in Patients with Cardiac Failure. Arq Bras Cardiol. 2006;86(6):451-8.

31. Gravina CF, Grespan SM, Borges JL. Tratamento não-medicamentoso da hipertensão no idoso. Rev Bras Hipertens. 2007;14(1):33-6.

32. Fialho PH, Tura BR, Sousa AS, Oliveira CR, Soares CCS, Oliveira JR et al. Effects of an exercise program on the functional capacity of patients with chronic Chagas heart disease, evaluated by cardiopulmonary testing. Rev Soc Bras Med Tropical. 2012;45(2):220-4.

33. Laterza MC, Rondon MUPB, Negrão CE. Efeito anti-hipertensivo do exercício. Rev Bras Hipertens, 2007;14(2):104-11.

34. Monteiro HL, Rolim LMC, Squinca DA, Silva FC, Ticianeli CCC, Amaral SL. Efetividade de um programa de exercícios no condicionamento físico, perfil metabólico e pressão arterial de pacientes hipertensos. Rev Bras Med do Esporte. 2007;13(2):107-12.

35. Winkelmann ER, Chiappa GR, Lima COC, Viecilli RRN, Stein R, Ribeiro JP. Addition of inspiratory muscle training to aerobic training improves cardiorespiratory responses to exercise in patients with heart failure and inspiratory muscle weakness. Am Heart J. 2009;158(5):768.e1-768.e7.

36. Nilson BB, Hellesnes B, Westhein A, Risberg MA. Group - based aerobic interval training in patients with chronic Heart Failure. Norwegian Ullevaal Model. Physical Therapy. 2008;88(4):530-5.

37. Vona M, Codeluppi GM, lannino T, Ferrari G, Bogousslavsky J, Von Segesser LK. Effects of different types of exercise training followed by detraining on endothelium-dependent dilation in patients with recent myocardial infarction. Circulation. 2009;119(12):1601-8

38. Rondon MUPB, Brum PC. Exercício físico como tratamento não farmacológico da hipertensão arterial. Rev Bras Hipertens. 2003;10(2):134-9.

39. Brum PC, Rondon MUPB, Silva GJJ, Krieger EM. Hipertensão arterial e exercício físico aeróbio. In: Negrão CE, Barretto ACP. Cardiologia do exercício: do atleta ao cardiopata. Barueri - SP: Manole, 2005;167-78

40. Cardoso J, Novaes M, Ochiai M, Regina K, Morgado P, Munhoz R, et al. Cardiomiopatia chagásica: prognóstico no perfil clínico-hemodinâmico C. Arq Bras Cardiol. 2010;95(4):518-23. 